\title{
On the effect of risk aversion in bimatrix games
}

Citation for published version (APA):

Berden, C., \& Peters, H. J. M. (2005). On the effect of risk aversion in bimatrix games. METEOR, Maastricht University School of Business and Economics. METEOR Research Memorandum No. 030 https://doi.org/10.26481/umamet.2005030

Document status and date:

Published: 01/01/2005

DOI:

10.26481/umamet.2005030

Document Version:

Publisher's PDF, also known as Version of record

\section{Please check the document version of this publication:}

- A submitted manuscript is the version of the article upon submission and before peer-review. There can be important differences between the submitted version and the official published version of record.

People interested in the research are advised to contact the author for the final version of the publication, or visit the DOI to the publisher's website.

- The final author version and the galley proof are versions of the publication after peer review.

- The final published version features the final layout of the paper including the volume, issue and page numbers.

Link to publication

\footnotetext{
General rights rights.

- You may freely distribute the URL identifying the publication in the public portal. please follow below link for the End User Agreement:

www.umlib.nl/taverne-license

Take down policy

If you believe that this document breaches copyright please contact us at:

repository@maastrichtuniversity.nl

providing details and we will investigate your claim.
}

Copyright and moral rights for the publications made accessible in the public portal are retained by the authors and/or other copyright owners and it is a condition of accessing publications that users recognise and abide by the legal requirements associated with these

- Users may download and print one copy of any publication from the public portal for the purpose of private study or research.

- You may not further distribute the material or use it for any profit-making activity or commercial gain

If the publication is distributed under the terms of Article $25 \mathrm{fa}$ of the Dutch Copyright Act, indicated by the "Taverne" license above, 


\title{
On the effect of risk aversion in bimatrix games
}

\author{
Caroline Berden* Hans Peters*
}

August 2005

\begin{abstract}
Nash equilibria with identical supports are compared for bimatrix games that are different with respect to the risk aversion of player 2 . For equilibria in $2 \times 2$-bimatrix games and for equilibria with efficient supports in coordination games it is established for which cases increased risk aversion of player 2 benefits or hurts player 2 .
\end{abstract}

\section{Introduction}

Uncertainty plays a central role in the theory of games, be it uncertainty about the parameters of the game - called incomplete information - or uncertainty about the actions of the opponent(s) - called strategic uncertainty by von Neumann and Morgenstern (1944). Following these authors, uncertainty is usually modelled by assuming the players to maximize expected utility. It is somewhat of a surprise that the effects of one of the most extensively studied characteristics of expected utility, namely the Arrow-Pratt measure of risk aversion (Arrow, 1971; Pratt, 1964), have received relatively little attention in game theory, with the exception of bargaining theory (starting with Kannai, 1977, and Kihlstrom et al., 1981).

In the present paper we study the effects of increased risk aversion in two-person noncooperative games with finite pure strategy sets, i.e., bimatrix games. Specifically, we consider the situation where player 2 is replaced

*Department of Quantitative Economics, University of Maastricht, P.O. Box 616, 6200 MD Maastricht, The Netherlands. Tel.: +31 43 3883835, email: c.berden@ke.unimaas.nl, h.peters@ke.unimaas.nl. 
by a more risk averse player and study the effect of this on Nash equilibrium. In doing so we assume that the supports of the Nash equilibria under comparison in the two situations - one with the less risk averse player 2 and the other one with the more risk averse player 2 - do not change, in order to make a meaningful comparison. This implies that the equilibrium strategy of player 2 and therefore the expected payoff of player 1 does not change. This may appear peculiar but it is a direct consequence of the fact that in a mixed strategy Nash equilibrium a player is indifferent between the pure strategies that are played with positive probability. Since it is meaningless to compare the expected payoffs of player 2 in the two situations, we compare the equilibria themselves. Specifically, we say that risk aversion benefits player 2 if the Nash equilibrium in the situation with the more risk averse player 2 would give the less risk averse player 2 a higher payoff. In the opposite case, we say that risk aversion hurts player 2 .

We give a complete analysis of the $2 \times 2$-case (Section 3 ), and of coordination games in which the players coordinate on Nash equilibria with efficient supports (Section 4 ). The analysis of the $2 \times 2$-case suggests that it is difficult to obtain results for the completely general case.

We identify cases where risk aversion benefits player 2-this happens in particular for Nash equilibria with efficient supports in coordination gamesand cases where risk aversion hurts player 2. In the former case, as an alternative interpretation, it would be advantageous for player 2 if he could make player 1 believe that he is more risk averse than he actually is. In the latter case, in contrast, player 2 would want player 1 to believe that he is less risk averse than he actually is. See also the discussion in Section 2.

After preliminaries in Section 2 we proceed with $2 \times 2$ games in Section 3 and coordination games in Section 4 . Section 5 concludes.

\section{Preliminaries}

A bimatrix game is a pair $(A, B)$ of $m \times n$-matrices of real numbers. A (mixed) strategy for player 1 is an element $p \in \Delta^{m}:=\left\{x \in \mathbb{R}^{m} \mid x \geq 0, \sum_{i=1}^{m} x_{i}=1\right\}$. Similarly, a strategy for player 2 is an element $q \in \Delta^{n}$. The strategy profile $(p, q)$ results in the expected payoffs $p A q$ for player 1 and $p B q$ for player 2 . A Nash equilibrium is a strategy profile $\left(p^{*}, q^{*}\right)$ such that $p^{*} A q^{*} \geq p A q^{*}$ for all $p \in \Delta^{m}$ and $p^{*} B q^{*} \geq p^{*} B q$ for all $q \in \Delta^{n}$. Let $a_{i j}\left(b_{i j}\right)$ denote the element in row $i$ and column $j$ of matrix $A(B)$. As is well known, $\left(p^{*}, q^{*}\right)$ is a Nash 
equilibrium if and only if

$$
\sum_{j=1}^{n} a_{i j} q_{j}^{*} \geq \sum_{j=1}^{n} a_{k j} q_{j}^{*} \text { for all } i \text { with } p_{i}^{*}>0 \text { and all } k=1, \ldots, m
$$

and

$$
\sum_{i=1}^{m} b_{i j} p_{i}^{*} \geq \sum_{i=1}^{m} b_{i k} p_{i}^{*} \text { for all } j \text { with } q_{j}^{*}>0 \text { and all } k=1, \ldots, n \text {. }
$$

Let $(A, B)$ be a bimatrix game and let $k$ be a concave and strictly increasing function defined on (at least) the interval $\left[\min \left\{b_{i j}\right\}, \max \left\{b_{i j}\right\}\right]$. Let $k(B)$ denote the matrix with element $k\left(b_{i j}\right)$ in row $i$ and column $j$. We say that player 2 in the game $(A, k(B))$ is more risk averse than player 2 in the game $(A, B)$ (cf. Pratt, 1964).

Suppose, in this situation, that $\left(p^{*}, q^{*}\right)$ is a Nash equilibrium in $(A, B)$ and that $\left(\tilde{p}, q^{*}\right)$ is a Nash equilibrium in $(A, k(B))$ such that $p^{*}$ and $\tilde{p}$ have the same support, that is, $p_{i}^{*}>0 \Leftrightarrow \tilde{p}_{i}>0$ for all $i=1, \ldots, m$. We say that risk aversion benefits player 2 at $\left(p^{*}, q^{*}\right)$ and $\left(\tilde{p}, q^{*}\right)$ if

$$
\tilde{p} B q^{*} \geq p^{*} B q^{*}
$$

and that risk aversion hurts player 2 at $\left(p^{*}, q^{*}\right)$ and $\left(\tilde{p}, q^{*}\right)$ if

$$
\tilde{p} B q^{*} \leq p^{*} B q^{*}
$$

In words, if risk aversion benefits [hurts] player 2, then the equilibrium in the game with the more risk averse player 2 is better [worse] for the less risk averse player 2 than the equilibrium in his own game.

Note that we assume that the two Nash equilibria under comparison are similar in the sense that their supports are the same. Hence, the players agree on the strategies that should be played with positive probability. This implies that player 2 should not (or, at least, must not) change his strategy ( $q^{*}$ in both equilibria), so that $(1)$ is fulfilled in both $(A, B)$ and $(A, k(B))$. Player 1 , however, adapts his strategy from $p^{*}$ in $(A, B)$ to $\tilde{p}$ in $(A, k(B))$, in order for (2) to hold with $\tilde{p}$ and $k(B)$ instead of $p^{*}$ and $B$.

Also note that it does not make sense to compare the expected payoffs $p^{*} B q^{*}$ and $\tilde{p} k(B) q^{*}$, since the numbers in $B$ and $k(B)$ represent the underlying preferences only up to a positive affine transformation. 
An alternative justification for definitions (3) and (4) is that player 1 may have only limited information about the utility function of player 2 . Specifically, suppose that player 1 knows player 2's utility function up to an increasing concave transformation, i.e., up to player 2's risk attitude. To fix ideas, one might think of the matrices $B$ and $k(B)$ as representing the utilities of monetary outcomes. Player 1 knows these monetary outcomes but not the utilities attached to them by player 2 . The inequalities in (3) and (4) reflect the possible consequences for player 2 in a Nash equilibrium if player 1 believes that player 2 is more risk averse than he actually is. For this interpretation we must implicitly assume that player 2 behaves myopically in the sense that he does not react optimally to the 'wrong' strategy of player 1 , that is, he does not play a strategy $\tilde{q}$ that maximizes his payoff $\tilde{p} B q$ over all $q \in \Delta^{n}$. Allowing for this possibility would only increase the effect in (3), but might undo the effect in (4). This assumption of myopic behavior makes sense if player 2 does not know that player 1 misjudges player 2's risk attitude. But even if player 2 tries to make player 1 believe that he (player 2 ) is more risk averse than he actually is - because risk aversion may benefit player 2-then he might still behave myopically in order not to reveal his true risk attitude, although making this argument precise would call for a dynamic model such as a repeated game.

The purpose of this note is to investigate in which cases risk aversion benefits or hurts player 2 . In the next section we analyze the $2 \times 2$-case, and in Section 4 we consider the special case of coordination games. As the $2 \times 2$-case will reveal, a complete analysis of the general case does not seem to be tractable.

\section{$32 \times 2$-bimatrix games}

We consider bimatrix games $(A, B)$ with $m=n=2$. Let $k$ be a strictly increasing concave transformation. For pure strategy Nash equilibria $\left(p^{*}, q^{*}\right)$ in $(A, B)$ we have, in the notation of the preceding section, $\tilde{p}=p^{*}$, so that (3) and (4) are fulfilled with equality. This case is not interesting and, in fact, we will assume, a forteriori, that player 2 has no (weakly) dominated strategy. For

$$
B=\left[\begin{array}{ll}
b_{11} & b_{12} \\
b_{21} & b_{22}
\end{array}\right]
$$


this implies that $b_{11} \neq b_{12}$. Without loss of generality we take $b_{11}>b_{12}$ and, thus, $b_{21}<b_{22}$. It is easy to verify that the following - not completely disjoint - cases are exhaustive:

(i) $b_{11}>b_{12} \geq b_{22}>b_{21}$;

(ii) $b_{11} \geq b_{22} \geq b_{12} \geq b_{21}$ and $b_{11}>b_{12}, b_{22}>b_{21}$;

(iii) $b_{11} \geq b_{22}>b_{21} \geq b_{12}$.

Let $\left(p^{*}, q^{*}\right)$ be a completely mixed Nash equilibrium in $(A, B)$, that is $0<$ $p_{1}^{*}, q_{1}^{*}<1$. (Of course, the existence of such an equilibrium depends on $A$ as well, but will just be assumed here.) Then

$$
p_{1}^{*}=\frac{b_{22}-b_{21}}{b_{22}-b_{21}+b_{11}-b_{12}}
$$

as can easily be verified.

We first consider case (i), and normalize the function $k$ such that $k\left(b_{21}\right)=$ $b_{21}$ and $k\left(b_{11}\right)=b_{11}$. Hence, $k\left(b_{22}\right) \geq b_{22}$ and $k\left(b_{12}\right) \geq b_{12}$ by concavity of $k$. For the equilibrium $(\tilde{p}, q)$ in $(A, k(B))$ we now derive

$$
\tilde{p}_{1}=\frac{k\left(b_{22}\right)-b_{21}}{k\left(b_{22}\right)-b_{21}+b_{11}-k\left(b_{12}\right)} \geq p_{1}^{*} .
$$

Hence

$$
\begin{aligned}
\tilde{p} B q^{*} & =\tilde{p}_{1}\left[q_{1}^{*} b_{11}+q_{2}^{*} b_{12}\right]+\tilde{p}_{2}\left[q_{1}^{*} b_{21}+q_{2}^{*} b_{22}\right] \\
& \geq p_{1}^{*}\left[q_{1}^{*} b_{11}+q_{2}^{*} b_{12}\right]+p_{2}^{*}\left[q_{1}^{*} b_{21}+q_{2}^{*} b_{22}\right] \\
& =p^{*} B q^{*}
\end{aligned}
$$

where the inequality follows from (6) and the fact that $q_{1}^{*} b_{11}+q_{2}^{*} b_{12} \geq q_{1}^{*} b_{21}+$ $q_{2}^{*} b_{22}$. We conclude that, in case (i), risk aversion benefits player 2 at $\left(p^{*}, q^{*}\right)$ and $\left(\tilde{p}, q^{*}\right)$.

Consider next case (ii). The analysis of case (i) goes through including (6), but now there are two cases:

(a) $q_{1}^{*} b_{11}+q_{2}^{*} b_{12} \geq q_{1}^{*} b_{21}+q_{2}^{*} b_{22}$. In this case, risk aversion again benefits player 2 , just like in case (i), see ( 7 ). 
(b) $q_{1}^{*} b_{11}+q_{2}^{*} b_{12} \leq q_{1}^{*} b_{21}+q_{2}^{*} b_{22}$. In this case we have the reverse inequality in (7), so that risk aversion hurts player 2 .

So in case (ii), whether risk aversion benefits or hurts player 2 depends on $q^{*}$, hence on the matrix $A$. Since

$$
q_{1}^{*}=\frac{a_{22}-a_{12}}{a_{22}-a_{12}+a_{11}-a_{21}}
$$

risk aversion benefits player 2 (case (a)) if

$$
\frac{a_{22}-a_{12}}{a_{22}-a_{12}+a_{11}-a_{21}} \geq \frac{b_{22}-b_{12}}{b_{22}-b_{12}+b_{11}-b_{21}}
$$

and risk aversion hurts player 2 (case (b)) if

$$
\frac{a_{22}-a_{12}}{a_{22}-a_{12}+a_{11}-a_{21}} \leq \frac{b_{22}-b_{12}}{b_{22}-b_{12}+b_{11}-b_{21}} .
$$

Consider, finally, case (iii). For this case we show by means of an example that the effect of risk aversion is ambiguous and depends not only on the matrices $A$ and $B$ but also on the function $k$. Consider the bimatrix games

$$
(A, B)=\left[\begin{array}{ll}
0,7 & 1,0 \\
1,2 & 0,4
\end{array}\right],\left(A, B^{\prime}\right)=\left[\begin{array}{ll}
0,5 & 1,0 \\
1,2 & 0,4
\end{array}\right],\left(A, B^{\prime \prime}\right)=\left[\begin{array}{cc}
0,7 & 1,-1 \\
1,2 & 0,4
\end{array}\right] .
$$

Clearly, both $B^{\prime}$ and $B^{\prime \prime}$ can be obtained by applying increasing concave transformations to $B$. The (unique) Nash equilibrium in $(A, B)$ is $\left(p^{*}, q^{*}\right)=$ $\left(\left(\frac{2}{9}, \frac{7}{9}\right),\left(\frac{1}{2}, \frac{1}{2}\right)\right)$, in $\left(A, B^{\prime}\right)$ it is $\left(p^{\prime}, q^{*}\right)=\left(\left(\frac{2}{7}, \frac{5}{7}\right),\left(\frac{1}{2}, \frac{1}{2}\right)\right)$, and in $\left(A, B^{\prime \prime}\right)$ it is $\left(p^{\prime \prime}, q^{*}\right)=\left(\left(\frac{1}{5}, \frac{4}{5}\right),\left(\frac{1}{2}, \frac{1}{2}\right)\right)$. Then $p^{*} B q^{*}=3 \frac{1}{9}, p^{\prime} B q^{*}=3 \frac{1}{7}$, and $p^{\prime \prime} B q^{*}=3 \frac{1}{10}$. Hence, risk aversion benefits player 2 at $\left(p^{*}, q^{*}\right)$ and $\left(p^{\prime}, q^{*}\right)$ but hurts player 2 at $\left(p^{*}, q^{*}\right)$ and $\left(p^{\prime \prime}, q^{*}\right)$.

The intuition for the different results in these three cases is as follows. In case (i), player 1 has to put more weight on the upper row in order to keep the more risk averse player 2 indifferent, but this row is unambiguously the best row from the point of view of player 2, so risk aversion benefits player 2 . In case (ii) player 1 again has to put more weight on the first row, but now it depends on $q^{*}$, and thus on the matrix $A$, whether the first or second row is better from the point of view of player 2 . This results in the two cases (a) and (b). In case (iii) it is not only ambiguous which one of the two rows is 
better from the point of view of player 2 , but also the change in weight put by player 1 on the first row is ambiguous, as is clear from the example.

We summarize and slightly extend these results in the following two propositions. The first proposition summarizes cases (i) and (ii).

Proposition 1 Let $m=n=2$, let $(A, B)$ and $(A, k(B))$ be bimatrix games with $k$ an increasing and concave function, and let $\left(p^{*}, q^{*}\right)$ and $\left(\tilde{p}, q^{*}\right)$ be completely mixed Nash equilibria in $(A, B)$ and $(A, k(B))$, respectively.

(i) If $b_{11}>b_{12} \geq b_{22}>b_{21}$, then risk aversion benefits player 2 at $\left(p^{*}, q^{*}\right)$ and $\left(\tilde{p}, q^{*}\right)$.

(ii) If $b_{11} \geq b_{22} \geq b_{12} \geq b_{21}$ and $b_{11}>b_{12}, b_{22}>b_{21}$, then risk aversion benefits player 2 at $\left(p^{*}, q^{*}\right)$ and $\left(\tilde{p}, q^{*}\right)$ if (8) holds and risk aversion hurts player 2 at $\left(p^{*}, q^{*}\right)$ and $\left(\tilde{p}, q^{*}\right)$ if $(9)$ holds.

The second proposition considers case (iii).

Proposition 2 Let $m=n=2$, let $(A, B)$ be a bimatrix game, and let $\left(p^{*}, q^{*}\right)$ be a completely mixed Nash equilibrium. Let $b_{11} \geq b_{22}>b_{21} \geq b_{12}$. Then there are increasing concave transformations $k$ and $k^{\prime}$ and Nash equilibria $\left(\tilde{p}, q^{*}\right)$ in $(A, k(B))$ and $\left(\tilde{p}^{\prime}, q^{*}\right)$ in $\left(A, k^{\prime}(B)\right)$ such that risk aversion benefits player 2 at $\left(p^{*}, q^{*}\right)$ and $\left(\tilde{p}, q^{*}\right)$ but hurts player 2 at $\left(p^{*}, q^{*}\right)$ and $\left(\tilde{p}^{\prime}, q^{*}\right)$.

Proof. Let $k$ satisfy $k(x)=x$ for all $x \leq b_{22}$ and $k\left(b_{11}\right)=b_{22}+\frac{1}{2}\left(b_{11}-b_{22}\right)$. Then it is straightforward to verify (using (5)) that $\tilde{p}_{1} \geq p_{1}^{*}$. Let $k^{\prime}$ satisfy $k^{\prime}(x)=x$ for all $x \geq b_{21}$ and $k^{\prime}\left(b_{12}\right)=b_{12}-\frac{1}{2}\left(b_{21}-b_{12}\right)$. Then it is easy to verify (again using (5)) that $\tilde{p}_{1}^{\prime} \leq p_{1}^{*}$. If (8) holds, then $k$ and $k^{\prime}$ are as desired. If (9) holds, then the proof is complete by switching the roles of $k$ and $k^{\prime}$.

Proposition 2 in particular makes it clear that there is not much hope to obtain general results for the $m \times n$-case. In the next section we consider the special but interesting case of coordination games.

\section{Coordination games}

A coordination game is an $m \times m$-bimatrix game $(A, B)$ such that $a_{i j}=b_{i j}=$ 0 whenever $i \neq j$, and $a_{i i}, b_{i i}>0$ for all $i=1, \ldots, m$. Clearly, the players 
would want to coordinate on one or more diagonal elements. In such a game, for each non-empty subset $I \subseteq\{1, \ldots, m\}$ there is a unique Nash equilibrium with support $I$ for each player, namely the strategy profile $\left(p^{I}, q^{I}\right)$ satisfying

$$
p_{i}^{I} b_{i i}=p_{j}^{I} b_{j j} \text { and } q_{i}^{I} a_{i i}=q_{j}^{I} a_{j j} \text { for all } i, j \in I, p_{k}=q_{k}=0 \text { for all } k \notin I \text {. }
$$

Observe that, for $m=2$, such a coordination game falls under case (ii) and under case (iii) in Section 3. In fact, it is a boundary case between these two: Proposition 2 applies, but the function $k^{\prime}$ defined in its proof is constant, so that it only depends on $q^{*}$ (i.e., on $A$ ) whether risk aversion benefits or hurts player 2 .

In a coordination game equilibria of interest are those where inefficient diagonal outcomes are played with zero probability. More generally, we say that a Nash equilibrium $\left(p^{I}, q^{I}\right)$ has efficient support if for all $i, j \in I, a_{i i}>$ $a_{j j}$ implies $b_{i i}<b_{j j}{ }^{1} \quad$ Clearly, if $\left(p^{I}, q^{I}\right)$ has efficient support and $k$ is an increasing concave function with $k(0)=0$, then the equilibrium $\left(\tilde{p}^{I}, q^{I}\right)$ in $(A, k(B))$ also has efficient support. ${ }^{2}$ Moreover, we have the following result.

Proposition 3 Let $(A, B)$ be an $m \times m$-coordination game and let, for some $I \subseteq\{1, \ldots, m\},\left(p^{I}, q^{I}\right)$ be a Nash equilibrium with efficient support in $(A, B)$. Let $k$ be an increasing concave function with $k(0)=0$ and let $\left(\tilde{p}^{I}, q^{I}\right)$ be a Nash equilibrium in $(A, k(B))$. Then risk aversion benefits player 2 at $\left(p^{I}, q^{I}\right)$ and $\left(\tilde{p}^{I}, q^{I}\right)$.

Proof. Without loss of generality suppose $I=\{1, \ldots, m\}$ and $0<b_{11} \leq$ $\ldots \leq b_{m m}$. We write $p$ instead of $p^{I}, \tilde{p}$ instead of $\tilde{p}^{I}$, and $q$ instead of $q^{I}$.

Since $(p, q)$ has efficient support and by $(10)$, we have $0<q_{1} \leq \ldots \leq q_{m}$, so that

$$
0<q_{1} b_{11} \leq \ldots \leq q_{m} b_{m m} .
$$

By concavity of $k, k\left(b_{i i}\right) / b_{i i} \geq k\left(b_{j j}\right) / b_{j j}$ whenever $i \leq j$. By (10) this implies

$$
\frac{\tilde{p}_{i}}{\tilde{p}_{j}}=\frac{k\left(b_{j j}\right)}{k\left(b_{i i}\right)} \leq \frac{b_{j j}}{b_{i i}}=\frac{p_{i}}{p_{j}} \text { for all } i, j \in I \text { with } i \leq j .
$$

\footnotetext{
${ }^{1}$ Clearly, unless $I$ is a singleton, such an equilibrium is neither ex ante nor ex post efficient. So the efficient support requirement is a very weak efficiency requirement.

${ }^{2}$ The condition $k(0)=0$ is imposed in order that $(A, k(B))$ is formally a coordination game. No generality is lost.
} 
For all $\ell, j \in I$ with $\ell>j,(12)$ implies

$$
\frac{p_{1}}{p_{\ell}}+\cdots+\frac{p_{j}}{p_{\ell}} \geq \frac{\tilde{p}_{1}}{\tilde{p}_{\ell}}+\cdots+\frac{\tilde{p}_{j}}{\tilde{p}_{\ell}}
$$

hence

$$
\frac{p_{\ell}}{p_{1}+\cdots+p_{j}} \leq \frac{\tilde{p}_{\ell}}{\tilde{p}_{1}+\cdots+\tilde{p}_{j}}
$$

hence

$$
\frac{p_{j+1}+\cdots+p_{m}}{p_{1}+\cdots+p_{j}} \leq \frac{\tilde{p}_{j+1}+\cdots+\tilde{p}_{m}}{\tilde{p}_{1}+\cdots+\tilde{p}_{j}}
$$

hence

$$
\frac{1-\left(p_{1}+\cdots+p_{j}\right)}{p_{1}+\cdots+p_{j}} \leq \frac{1-\left(\tilde{p}_{1}+\cdots+\tilde{p}_{j}\right)}{\tilde{p}_{1}+\cdots+\tilde{p}_{j}}
$$

which implies

$$
p_{1}+\cdots+p_{j} \geq \tilde{p}_{1}+\cdots+\tilde{p}_{j} \text { for all } j=1, \ldots, m .
$$

Define $b_{00}:=0$, then

$$
\begin{aligned}
\sum_{j=1}^{m} p_{j} q_{j} b_{j j} & =\sum_{j=1}^{m}\left(\sum_{i=j}^{m} p_{i}\right)\left(q_{j} b_{j j}-q_{j-1} b_{j-1, j-1}\right) \\
& \leq \sum_{j=1}^{m}\left(\sum_{i=j}^{m} \tilde{p}_{i}\right)\left(q_{j} b_{j j}-q_{j-1} b_{j-1, j-1}\right) \\
& =\sum_{j=1}^{m} \tilde{p}_{j} q_{j} b_{j j},
\end{aligned}
$$

where the inequality follows from (11) and (13). Now (14) shows that risk aversion benefits player 2 .

As a note to this proof, observe that (13) states that $\tilde{p}$ first-degree stochastically dominates $p$, and (14) is the familiar consequence that the expected payoff under $\tilde{p}$ is higher than under $p$.

The intuition for Proposition 3 is that increased risk aversion of player 2 forces player 1 to put more weight on the outcomes that are better for player 2. From the point of view of the original - less risk averse-player 2 the resulting distribution is preferable. This intuition (and result) is similar to the result established for a bargaining context in Köbberling and Peters (2003), although that paper assumes rank-dependent utility instead of expected utility. 


\section{Concluding remarks}

The present paper has shown that at least in some interesting cases it is possible to say something about the effect of the risk attitude of a player in a noncooperative game. From a more general point of view the paper can also be interpreted as studying the effect of one player having limited information about the utility function of another player. We focus, however, on risk attitude, which is one of the most important characteristics of an expected utility function. Further research may concentrate on specific economic games, such as risky investments in portfolios.

\section{References}

1. Arrow, K.J. (1971): Essays in the Theory of Risk Bearing. Markham, Chicago, Illinois.

2. Kannai, Y. (1977): "Concavifiability and constructions of concave utility functions," Journal of Mathematical Economics, 4, 1-56.

3. Kihlstrom, R.E., A.E. Roth, and D. Schmeidler (1981): "Risk aversion and solutions to Nash's bargaining problem," in: O. Moeschlin and D. Pallaschke (eds.), Game Theory and Mathematical Economics. North Holland, Amsterdam.

4. Köbberling, V., and H. Peters (2003): "The effect of decision weights in bargaining problems," Journal of Economic Theory, 110, 154-175.

5. Pratt, J.W. (1964): "Risk aversion in the small and in the large," Econometrica, 32, 122-136.

6. von Neumann, J., and O. Morgenstern (1944): Theory of Games and Economic Behavior. Princeton University Press, Princeton. 